\title{
THE USE OF A POSITION SENSITIVE DE- TECTOR OR OF A MULTIDETECTOR FOR THE MEASUREMENT OF POLE FIGURES BY NEUTRON TIME-OF-FLIGHT TECH- NIQUE
}

\section{K. WALTHER.}

Joint Institute for Nuclear Research, Laboratory for Neutron Physics, 141980 Dubna, USSR.

\section{Introduction}

The neutron flux of even high flux reactors is poor in comparison with the quantum flux of X-ray tubes. Furthermore, the costs of operation time of a reactor exceeds the ones of a X-ray diffractometer many times. Therefore in order to decrease the measuring time more and more neutron spectrometers have been equipped with multidetectors (MD) or position sensitive detectors (PSD) in the last time. Also in the field of texture investigation the use of PSD were reported. They are used at spectrometers working with monochromated neutron beams and are particularly used to do profile anulysis. In the present paper we describe the planned improvement of a spectrometer working with a white neutron beam (time-of-flight technique) using a bent MD. In its nature this is a combination of both wavelength scan and pole figure scan. For scanning the pole figures the sample movement can be reduced to a rotation about only one axis.

\section{Measurement with monochromatic neutrons}

A "classical" neutron diffraction experiment needs a monochromated neutron beam with a certain wavelength $\lambda_{0}$. According to BRAGG's law at a 
certain scattering angle $\vartheta$ the pole figure $\tilde{P}_{h k l}$ can be scanned step by step. The scanning is done by rotating the sample in an Eulerian cradle. This method is very time consuming, especially if a high number of pole figures are needed due to a low crystal symmetry. For investigation of dynamic processes this method would not be applicable.

The measuring time can be reduced by using a MD or a PSD. Up to now there have been reported two different methods for the use of $\mathrm{MD}$ or PSD in texture investigation by means of neutron diffraction. D. Juul Jensen [1] uses a linear PSD which covers a range direct in the pole figure. The number of rotation steps for scanning a pole figure is reduced considerably. This method requires a fixed scattering angle $2 \vartheta=90^{\circ}$ where the DEBYE-SCHERRER-cone for the scattered neutrons degenerate into a plane. The change from a pole figure to another one is managed by changing the wavelength $\lambda_{0}$ of the incident beam with a double monochromator.

Another proposal was made by Bunge et al. [2]. A bent MD was used for scanning in the $2 \vartheta$-scale. In this manner some pole figures can be measured simultaneously. In certain cases overlapped diffraction peaks may be separated by profile analysis. Scanning some pole figures simultaneously every detecting element (detector cell) of such a MD views at the sample under its own "pole figure angle". This means, the sampling mesh of the pole figures differ somewhat from another.

\section{Measurement with time-of-flight method}

Using the time-of-flight method for the measurement of pole figures $[3,4]$, the detector makes wavelength scans. Similar to the last mentioned method all pole figures are measured simultaneously; overlapped peaks can be separated by profile analysis. The sampling meshes are the same for all pole figures. Using two or more detectors - which are located in the horizontal plane and differing in the scattering angle from each other - two or more points in all pole figures are measured simultaneously. But all wavelengthdependent corrections as absorption, extinction and correction for the intensity of the incident beam must be done for each detector separately. Also the resolution will vary from detector to detector.

These disadvantages can be avoided by an arrangement of the detectors on a DFBYE-SCHERRER-cone so that all detector cells see the sample under the same scattering angle 2v. The principal layout of such an arrangement is shown in fig. 1. The detecting device may be a PSD bent into a circular arc or may be a MD, which elements form a circular arc. The scattering angle $2 \vartheta$ can widely be changed by varying the distance $d$ from the sample to the centre of the arc. With $r$ as the radius of the arc we yield $\tan \vartheta=d / r$. 


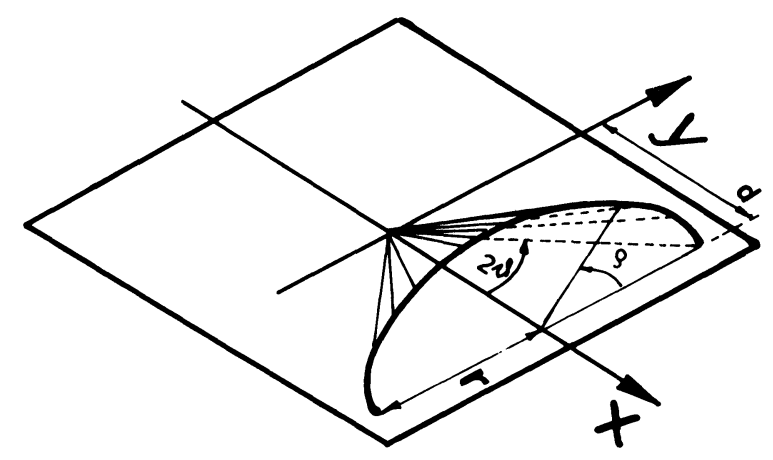

Figure 1: Principal layout of a bent detecting device. The angle $\rho$ denotes the cell or the position.
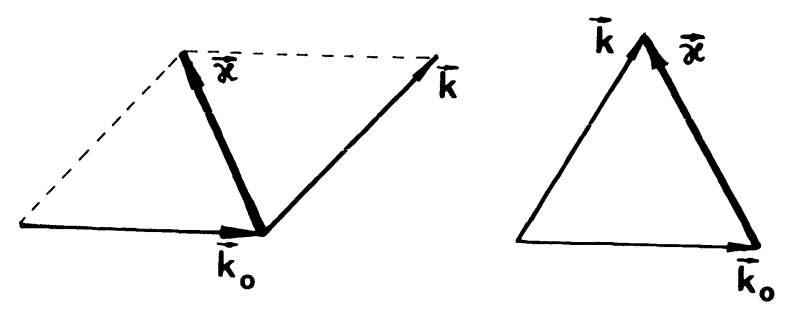

Figure 2: Definition of the vectors $\vec{k}, \vec{k}_{0}$, and $\vec{k}$

We describe the position along the circumference of the position sensitive detector or of the cell of the MD by an angle $\rho$ varying from 0 to $\pi$.

Mathematically we describe the elastic scattering with the vectors $\vec{k}_{0}$, $\vec{k}$, and $\vec{k}$. In fig. 2 these vectors are schematically shown. The direction of the incident beam and the direction of the scattered bearn are represented by the vectors $\vec{k}_{0}$ and $\vec{k}$, respectively. The vector $\vec{k}$ depends on the angles $\vartheta$ and $\rho^{1}$ :

$$
\vec{k}=\left(\begin{array}{c}
\cos 2 \vartheta \\
\sin 2 \vartheta \cos \rho \\
\sin 2 \vartheta \sin \rho
\end{array}\right)
$$

The difference $\vec{k}=\vec{k}-\vec{k}_{0}$ is the scattering vector. Its direction is always normal to the scattering net plane.

If a PSD or a MD is used, each cell or position will be associated with

\footnotetext{
'Here and in the following ell vectork are normalised.
} 


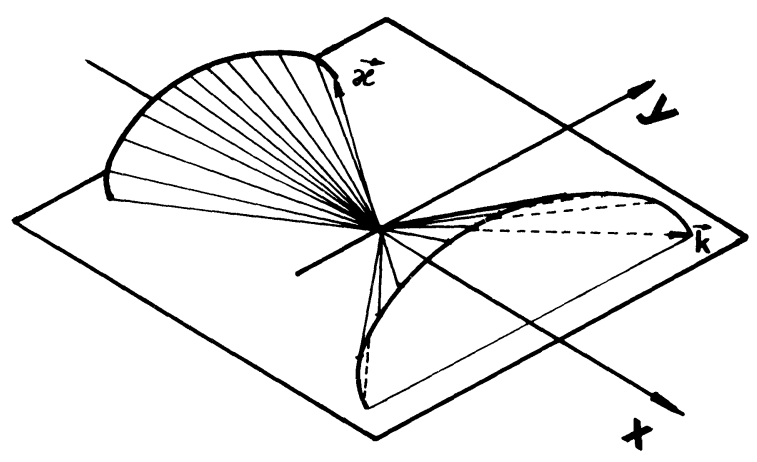

Figure 3: Changing the angle $\rho$ the vector $\vec{k}$ describes a cone

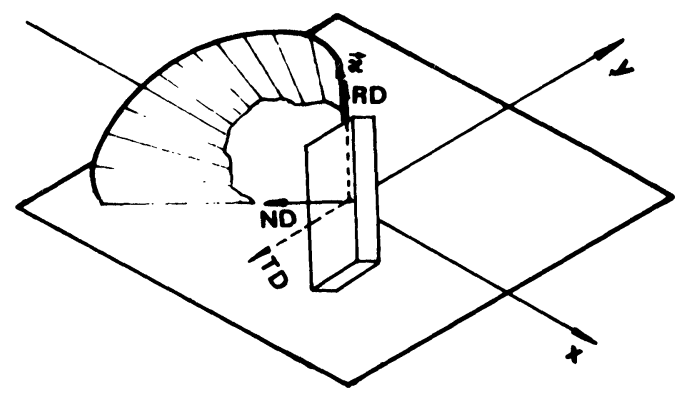

Figure 4: Orientation of the sample coordinate system $K_{A}$ in the laboratory coordinate system $K_{L}$ for symmetric transmission

its own scattering vector, dependent on $\vartheta$ and $\rho$.

$$
\vec{R}=\left(\begin{array}{c}
-\sin \vartheta \\
\cos \vartheta \cos \rho \\
\cos \vartheta \sin \rho
\end{array}\right)
$$

Changing $\rho$ for a fixed scattering angle $\vartheta$ the scattering vector describe also a cone of semi-angle $\frac{\pi}{2}-\vartheta$ (see fig. 3 ). In the case of back scattering this cone will be degenerate into a straight.

Now let us put a sample with its own coordinate system (RD,TD and ND) into a goniometer, choosing the symmetric transmission case. (See fig. 4.)

In the laboratory coordinate system the sample axis TD points down in direction $-z$, where $R D$ and $N D$ lie in the $x-y$ plane with $R D \| \vec{k}_{0}^{2}$.

\footnotetext{
${ }^{2} x_{0}$ otende for $R(p=0)$.
} 
Laboratory coordinates $K_{L}$ can be translated into the sample coordinate system $K_{A}$ by the following transformation:

$$
K_{A}=\left(\begin{array}{ccc}
-\sin \vartheta & \cos \vartheta & 0 \\
0 & 0 & -1 \\
-\cos \vartheta & -\sin \vartheta & 0
\end{array}\right) \cdot K_{L}
$$

Each detector cell "sees" in the sample another net plane, i. e. another direction $\vec{y}$ within the pole figure. The polar coordinates $\theta$ and $\varphi$ are the polar coordinates of the associated scattering vector $\vec{k}(\vartheta, \rho)$ with respect to the sample coordinate system $K_{A}$.

The pole angle $\theta(\vartheta, \rho)$ is the angle between $\vec{\kappa}(\vartheta, \rho)$ and NR. We yield:

$$
\cos \theta(\vartheta, \rho)=\frac{1}{2} \sin 2 \vartheta(1-\cos \rho)
$$

For scattering angles $2 \vartheta<90^{\circ}$ you can only measure the part of the pole figure beginning from the equator till to a lower limit $\theta_{\min }(\vartheta)=$ arcoos $(\sin 2 \vartheta)$ with scanning in $\rho$. For $2 \vartheta=90^{\circ}$ (4) will reduce to

$$
\theta_{00}(\rho)=\sin ^{2} \rho / 2
$$

In this case the whole polar range of the pole figure can be measured scanning on $\rho$. Larger scattering angles will limit the range for the $\theta$-angle again.

The angle $\varphi$ also changes during the scan on $\rho$. We scale this angle starting at the rolling direction:

$$
\tan \varphi=\frac{-\cos \vartheta \sin \rho}{1+\cos ^{2} \vartheta(\cos \rho-1)}
$$

For $2 \vartheta=90^{\circ}$ this formula simplifies to

$$
\tan \varphi=-\frac{\sqrt{2} \sin \rho}{1+\cos \rho}
$$

The resulting sampling mesh for a fixed scattering angle $2 \vartheta^{9}=90^{\circ}$ is shown in fig. 5. The scan on the angle $\rho$ is shown as emphasized points. In this example the step width in $\rho$ was chosen to be $10^{\circ}$. Turning the sample around its normal all pole figures are scanned simultaneously. The scanning of pole figures is reduced to rotation on only one axis.

\section{Summary}

In order to minimize the experimental effort for measuring pole figures it is proposed to use a position sensitive detecting device even for time-of-flight 


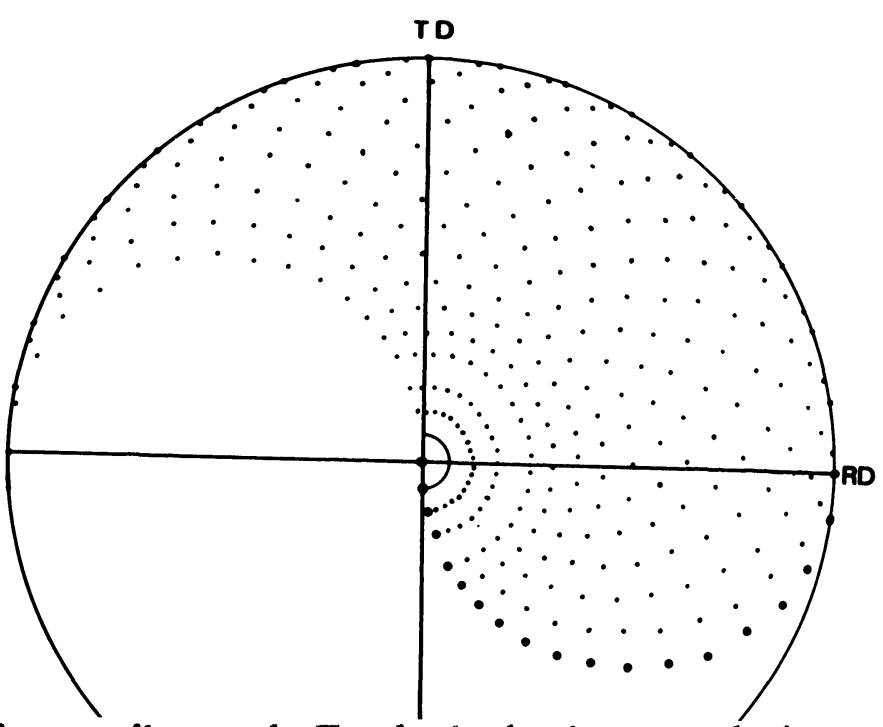

Figure 5: The sampling mesh. Emphasized points records the scan through $\rho, 2 \vartheta=90^{\circ}$

technique. In its nature this is a combination of both wavelength scan and pole figure scan. The geometric shape of this device should be a semicircle. This device should be set up parallel to the $y-z$ plane with his symmetry axis along the incident beam. Although there are not principal limitations for the scattering angle, an experimental layout with $2 \vartheta=90^{\circ}$ should be preferred. In this case the scan of the pole figures will be reduced to a rotation only on the normal of the sample. For these quick measurements a suitable sample geometry is a cube or a sphere. The dimension of the sample should be in the same order as the absorption half thickness (i. e. for neutrons few $\mathrm{cm}$ in the most cases).

\section{References}

[1] D. Juul Jensen, Proc. Workshop on Experimental Techniques of Texture Analysis, Ed. H.-J. Bunge, DGM Informationsgesellschaft, Oberursel, pp 217-228, 1985

[2] H.-J. Bunge et al., Textures and Microstructures, 5, pp 153-170, (1982)

[3] W. Matz et al., Report ZfK-452, ZfK Rosendorf, GDR, 1981

[4] K. Feldmann et al., Proc. Workshop on Theoretical Methods of Texture Analyais, Ed. H.-J. Bunge, DGM Informationsgesellschaft, Oberursel, pp 207-214, 1986 